\title{
Optimum Array Processing with Variable Linear Constraint
}

\author{
Byong Kun Chang*, Member, KIICE \\ Department of Electrical Engineering, University of Incheon, Incheon 406-772, Korea
}

\begin{abstract}
A general linearly constrained adaptive array is examined in the weight vector space to illustrate the array performance with respect to the gain factor. A narrowband linear adaptive array is implemented in a coherent signal environment. It is shown that the gain factor in the general linearly constrained adaptive array has an effect on the linear constraint gain of the conventional linearly constrained adaptive array. It is observed that a variation of the gain factor of the general linearly constrained adaptive array results in a variation of the distance between the constraint plane and the origin in the translated weight vector space. Simulation results are shown to demonstrate the effect of the gain factor on the nulling performance.
\end{abstract}

Index Terms: Adaptive array, Coherent, Gain Factor, Linear constraint, Narrowband, Sensor element

\section{INTRODUCTION}

If the desired signal is uncorrelated with incoming interference signals, a linearly constrained adaptive array successfully estimates the desired signal by reducing the interference signals [1]. If the desired signal is correlated partially or totally (i.e., coherent) with the interference signals, the desired signal is partially or totally cancelled in the array output depending on the extent of correlation between the desired signal and the interference signals.

Some methods, such as the spatial smoothing approach [2, 3], master-slave type array processing [4], alternate mainbeam nulling method [5], and general linearly constrained adaptive array [6], have been proposed to prevent the signal cancellation phenomenon in a correlated signal environment. A drawback of the methods proposed in [2-5] is that they employ additional hardware or algorithms to reduce the effect of the coherent interferences.

In this study, the general linearly constrained adaptive array is examined in the weight vector space to find the nulling performance in [6] in terms of the gain factor, which turns out to be the reduction of the gain in the look direction in the translated weight vector space. It is shown that the variation of the gain factor results in the variation of the distance between the constraint plane and the origin in the translated weight vector space, which has a geometric effect of shifting the constraint plane with respect to the origin.

The simulation results are shown to illustrate the nulling performance with respect to the gain factor. It is shown that the general linearly constrained adaptive array performs better than the linearly constrained adaptive array with respect to the elimination of the coherent interferences.

\section{OPTIMUM WEIGHT VECTOR}

It is assumed that a desired signal is incident from a known direction (i.e., the look direction) while coherent interferences come from unknown directions on the narrowband general linearly constrained adaptive array with $N$ sensor elements, as shown in Fig. 1. The weights $\boldsymbol{w}_{\boldsymbol{n}}, 1 \leq n \leq N$, are adjusted to find an optimum weight

Received 29 January 2014, Revised 14 February 2014, Accepted 25 April 2014

*Corresponding Author Byong Kun Chang (E-mail: chang@incheon.ac.kr, Tel: +82-32-770-8436)

Department of Electrical Engineering, University of Incheon, 119 Academy-ro, Yeonsu-gu, Incheon 406-772, Korea.

(c) This is an Open Access article distributed under the terms of the Creative Commons Attribution Non-Commercial License (http://creativecommons.org/li-censes/by$\mathrm{nc} / 3.0 /$ ) which permits unrestricted non-commercial use, distribution, and reproduction in any medium, provided the original work is properly cited. 


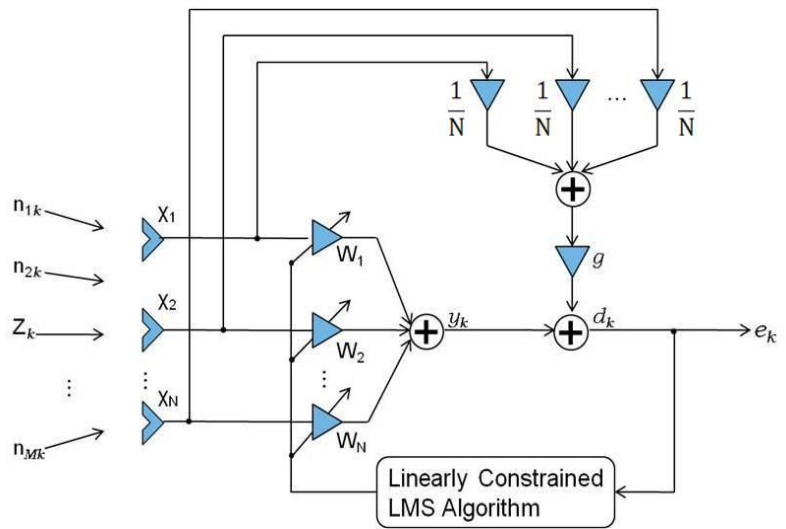

Fig. 1. Narrowband general linearly constrained adaptive array. LMS: least mean squares.

vector to estimate the desired signal with unit gain in the look direction. It is to be noted in the figure that the conventional beamformer output is multiplied by the gain factor $g$ to yield the desired response $d_{k}$.

To find the array weights that optimally estimate the desired signal in the look direction while eliminating the undesired interference signals as much as possible, we solve the following optimization problem in which the mean square error is minimized subject to the unit gain constraint in the look direction.

$$
\begin{gathered}
\min E\left[\left|e_{k}\right|^{2}\right], \\
\text { subject to } \boldsymbol{w}^{H} \mathbf{s}=1,
\end{gathered}
$$

where the error signal between the adaptive array output and the desired response is given by

$$
e_{k}=y_{k}-d_{k}
$$

the output of the adaptive array is represented as

$$
y_{k}=\boldsymbol{w}^{H} \boldsymbol{x}_{k}
$$

the desired response is given by

$$
d_{k}=g \frac{s^{H} x_{k}}{N}
$$

and the signal vector, the weight vector $\boldsymbol{w}$, and the steering vector $\boldsymbol{s}$ for the look direction are respectively given by

$$
\begin{aligned}
& \boldsymbol{x}_{k}=\left[\begin{array}{llll}
x_{1, k} & x_{2, k} & \cdots & x_{N, k}
\end{array}\right]^{T}, \\
& \boldsymbol{w}=\left[\begin{array}{llll}
w_{1} & w_{2} & \cdots & w_{N}
\end{array}\right]^{T}, \\
& \boldsymbol{s}=\left[\begin{array}{lllll}
1 & e^{-j \beta \tau_{0}} & e^{-j 2 \beta \tau_{0}} & \cdots & e^{-j(N-1) \beta \tau_{0}}
\end{array}\right]^{T},
\end{aligned}
$$

where $\beta$ denotes the radian frequency of the desired signal, $\tau_{0}=d \sin \theta_{0} / c, \theta_{0}$ indicates the incident angle of the desired signal from the array normal, $d$ refers to the interelement spacing, $c$ represents the signal propagation velocity, $k$ indicates the iteration index, and $E, T$, and $H$ denote the expectation, transpose, and complex conjugate transpose, respectively.

It can be shown that the mean squared error $E\left[\left|e_{k}\right|^{2}\right]$ is given by

$$
E\left[\left|e_{k}\right|^{2}\right]=\left(\boldsymbol{w}-g \frac{\mathbf{s}}{N}\right)^{\mathrm{H}} \boldsymbol{R}\left(\boldsymbol{w}-g \frac{\mathbf{s}}{N}\right),
$$

where

$$
\boldsymbol{R}=E\left[\boldsymbol{x}_{k} \boldsymbol{x}_{k}^{H}\right]
$$

The method of Lagrange multipliers [1] is used for finding the optimal solution by solving the unconstrained optimization problem with the objective function

$$
O(\boldsymbol{w})=\left(\boldsymbol{w}-g \frac{\mathbf{s}}{N}\right)^{\mathrm{H}} \boldsymbol{R}\left(\boldsymbol{w}-g \frac{\mathbf{s}}{N}\right)+\lambda\left(\boldsymbol{w}^{\mathrm{H}} \mathbf{s}-1\right),
$$

where $\lambda$ denotes a Lagrange multiplier.

Here, the optimum weight vector is given by

$$
\boldsymbol{w}_{\text {opt }}=g \frac{\mathbf{s}}{N}+(1-g) \frac{\boldsymbol{R}^{-1} \boldsymbol{s}}{s^{H} R^{-1} s} .
$$

It is observed in (11) that the optimum weight vector lies between the uniform weight of the conventional beamformer and the optimum weight vector for the unit gain constraint depending on the value of the gain factor.

\section{OPTIMUM WEIGHT VECTOR IN THE TRANSLATED WEIGHT VECTOR SPACE}

We designate the weight vector $\boldsymbol{w}$ translated by $g \frac{\boldsymbol{s}}{N}$ as $\boldsymbol{v}$. Then, the optimization problem in (1) is formulated in terms of $\boldsymbol{v}$ as

$\min \boldsymbol{v}^{H} \boldsymbol{R} \boldsymbol{v}$,

$$
\text { subject to } \boldsymbol{v}^{H} \mathbf{s}=1-g \text {. }
$$

Solving (12) by using the Lagrange multiplier method, we obtain the optimum weight vector as

$$
\boldsymbol{v}_{o p t}=(1-g) \frac{\boldsymbol{R}^{-1} \boldsymbol{s}}{\boldsymbol{s}^{H} R^{-1} \boldsymbol{s}} .
$$

The optimum weight vector in (13) is for the unit gain constraint scaled by $(1-g)$. 


\section{GENERAL LINEARLY CONSTRAINED LMS ALGORITHM}

The steepest descent method [7] is employed to find the iterative solution for the optimum weight vector, where the next weight vector is given by the current one added by the negative gradient with respect to $\boldsymbol{v}$ and scaled by a convergence parameter as follows:

$$
\boldsymbol{v}_{k+1}=\boldsymbol{v}_{k}+\mu\left(-\nabla_{v} \mathrm{O}(\boldsymbol{v})\right)
$$

where $\mu$ denotes the convergence parameter.

The objective function is given by

$$
\mathrm{O}(\boldsymbol{v})=\boldsymbol{v}^{\mathrm{H}} \boldsymbol{R} \boldsymbol{v}+\lambda\left(\boldsymbol{v}^{\mathrm{H}} \boldsymbol{s}-(1-g)\right)
$$

Applying the constraints in (12) to (14) to find the value of $\lambda$ and manipulating the resulting equation, we find the following iterative solution:

$$
\boldsymbol{v}_{k+1}=\left[\boldsymbol{I}-\frac{s \boldsymbol{s}^{H}}{N}\right]\left[\boldsymbol{v}_{k}-\mu \boldsymbol{R} \boldsymbol{v}_{k}\right]+(1-g) \frac{\boldsymbol{s}}{N},
$$

where

$$
\boldsymbol{v}_{k}=\left[\begin{array}{llll}
v_{1, k} & v_{2, k} & \cdots & v_{N, k}
\end{array}\right]^{T} .
$$

$\boldsymbol{I}$ denotes the $N \times N$ identity matrix. If $\boldsymbol{R}$ is estimated using an instantaneous approximation, i.e., $\boldsymbol{R}=\boldsymbol{x}_{k} \boldsymbol{x}_{k}^{H}$, a stochastic adaptive algorithm is derived; it can be represented as

$$
\boldsymbol{v}_{k+1}=\left[\boldsymbol{I}-\frac{s s^{H}}{N}\right]\left[\boldsymbol{v}_{k}-\mu e_{k}^{*} \boldsymbol{x}_{k}\right]+(1-g) \frac{s}{N},
$$

where $*$ denotes a complex conjugate.

Eq. (18) is called the general linearly constrained least mean square (LMS) algorithm. In (19), it is observed that the updated weight vector $\boldsymbol{v}_{k}$ is projected onto the constrained subspace, which is an orthogonal complement of the subspace spanned by the steering vector of the desired signal. Then, the projected weight vector is added by the scaled version of the steering vector for the look direction. Notice that the steering vector is orthogonal to the constrained plane.

Thus, a variation of the gain factor results in a variation of the distance between the constraint plane and the origin in the translated weight vector space (i.e., an increase in the gain factor results in a decrease in the distance). This phenomenon has an effect on the nulling performance of the general linearly constrained adaptive array in terms of the gain factor.

\section{SIMULATION RESULTS}

To illustrate the nulling performance of the general linearly constrained adaptive array in terms of the gain factor, the simulation results in [6] are redisplayed for the cases of one and two coherent interferences.

A narrowband linear array with 7 equispaced sensor elements is employed to examine the performance of the general linearly constrained adaptive array. The incoming signals are assumed to be plane waves. The desired signal is assumed to be a sinusoid incident on the linear array at the array normal. The cases for one and two coherent interferences are simulated. The nulling performances are compared with respect to the gain factor $g$ and with the linearly constrained adaptive array. The convergence parameter $\mu$ is assumed to be 0.001 .

\section{A. Case of One Coherent Interference Case}

It is assumed that a coherent interference is incident at $30^{\circ}$ from the array normal. The variation of the error power between the array output and the desired signal is displayed in terms of the gain factor $g$ in Fig. 2. The optimum value of $g$, which yields the minimum error power, is shown to be 0.331 . The comparison of the array performance for $g=0.331,0.01$, and the linearly constrained adaptive array is shown in Figs. 3 and 4 with respect to the array output and the desired signal for $k=1-1000$ and $29001-30000$ samples, respectively. It is demonstrated that the case for $g=0.331$ performs best while the case for $g=0.01$ performs better than the general linearly constrained adaptive array. It is observed that the desired signal of the linearly constrained adaptive array disappears (i.e., is cancelled out) in the array output for $29001 \leq k \leq 30000$

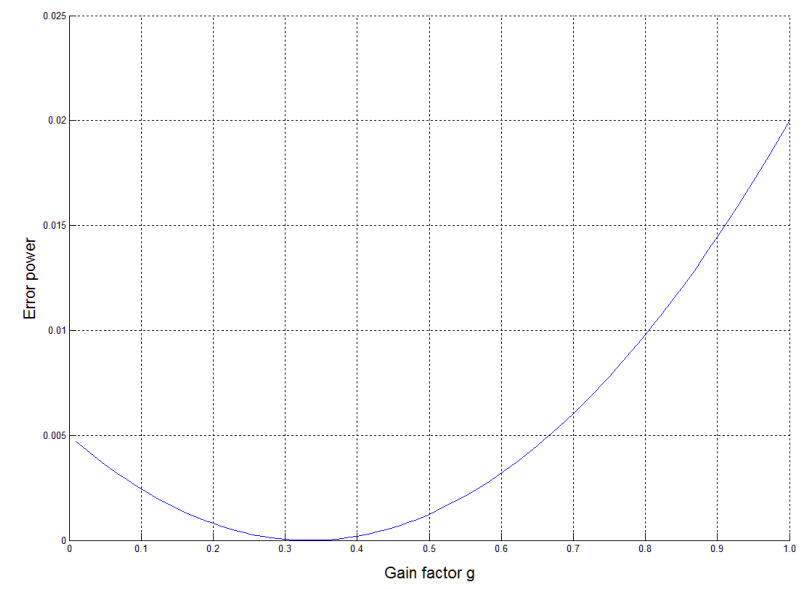

Fig. 2. Variation of the error power in terms of the gain factor for the case of one coherent interference. 

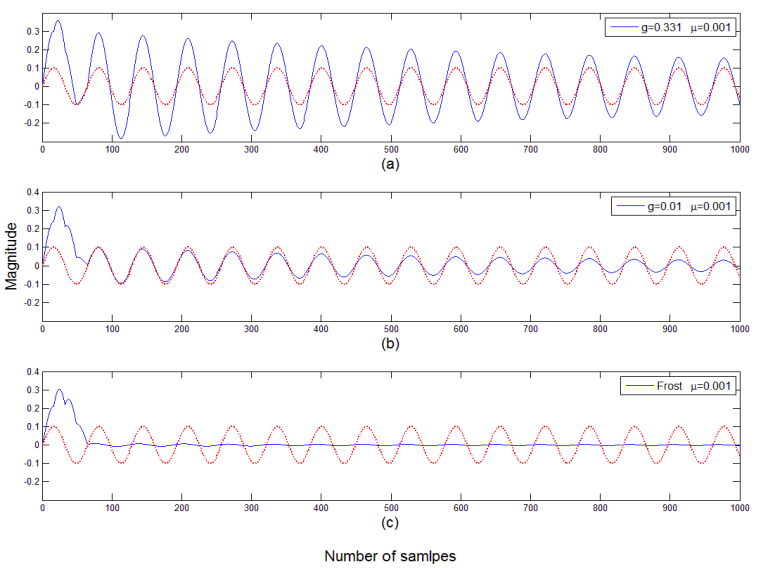

Fig. 3. Comparison of the array output and the desired signal for the case of one coherent interference for $1 \leq k \leq 1000$.
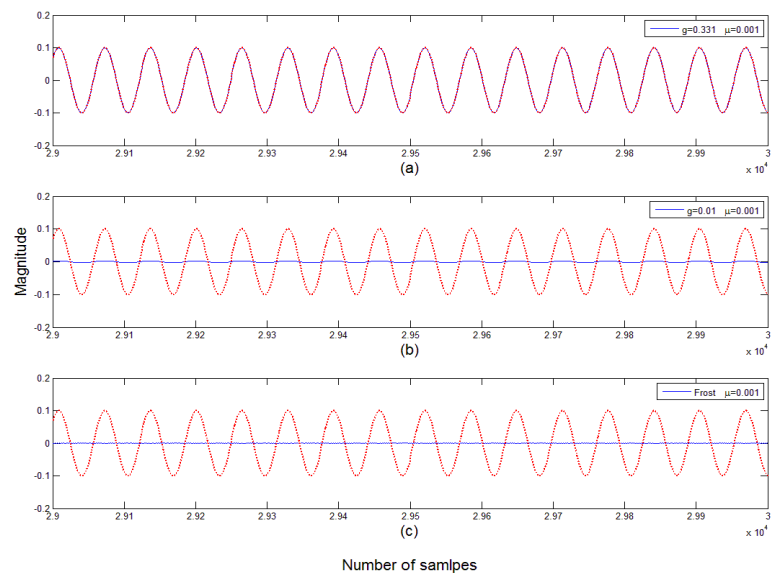

Fig. 4. Comparison of the array output and the desired signal for the case of one coherent interference for $29001 \leq k \leq 30000$.

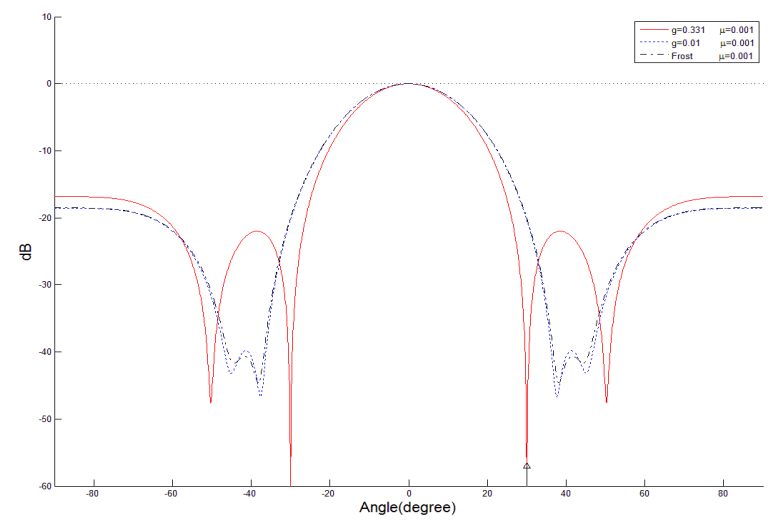

Fig. 5. Comparison of the beam patterns for the case of one coherent interference.

The beam patterns are shown in Fig. 5, in which the case for $g=0.331$ forms a deep null $(-51 \mathrm{~dB})$ in the direction of the interference $\left(30^{\circ}\right)$.

\section{B. Case of Two Coherent Interferences}

It is assumed that two coherent interferences are incident at $-54.3^{\circ}$ and $57.5^{\circ}$ from the array normal. The variation of the error power between the array output and the desired signal is displayed in Fig. 6. The optimum value of $g$ is shown to be 0.632 . The comparison of the array performances for $g=0.632,0.01$ and the linearly constrained adaptive array is shown in Figs. 7 and 8 with respect to the array output and the desired signal for $k=1-1000$ and $29001-30000$ samples, respectively. It is shown that the case for $g=0.632$ performs best, while the case for $g=0.01$ performs similar to the linearly constrained adaptive array. The beam patterns are shown in Fig. 9, in which the case for $g=0.632$ forms two deep nulls $(-36.6 \mathrm{~dB}$ and $-30.4 \mathrm{~dB})$ at the incident angles $\left(-54.3^{\circ}\right.$ and $57.5^{\circ}$ ) of the two coherent interferences, while the gains for the linearly constrained adaptive array are $-21.0 \mathrm{~dB}$ and $21.8 \mathrm{~dB}$ and the gains for $g=0.01$ are $-22.7 \mathrm{~dB}$ and -25.1 $\mathrm{dB}$, respectively.

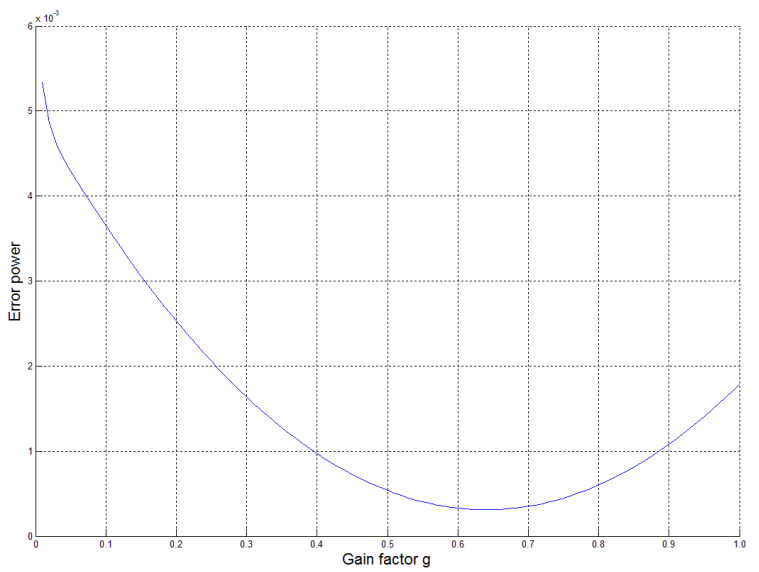

Fig. 6. Variation of the error power in terms of the gain factor for the case of two coherent interferences.
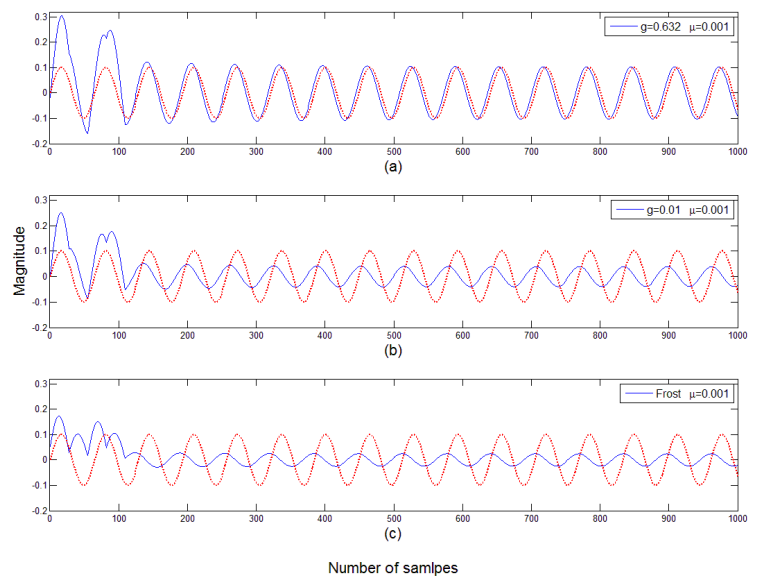

Fig. 7. Comparison of the array output and the desired signal for the case of two coherent interferences for $1 \leq k \leq 1000$. 

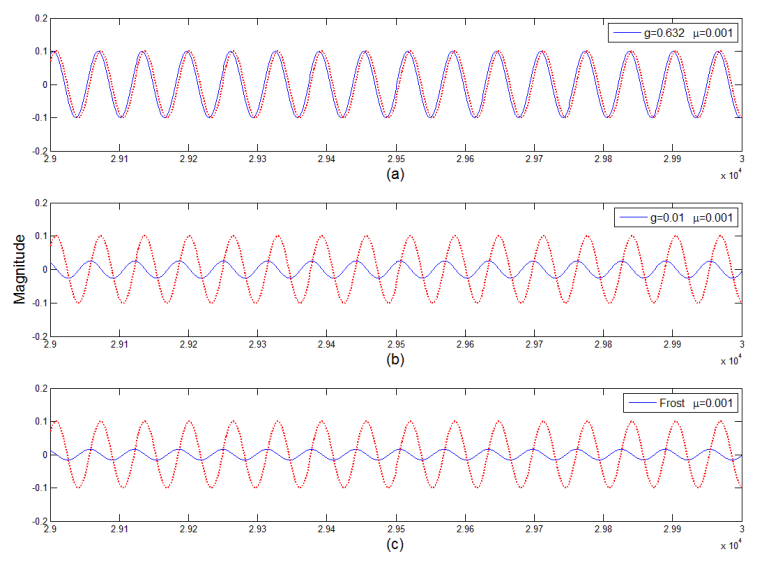

Number of samipes

Fig. 8. Comparison of the array output and the desired signal for the case of two coherent interferences for $29001 \leq k \leq 30000$.

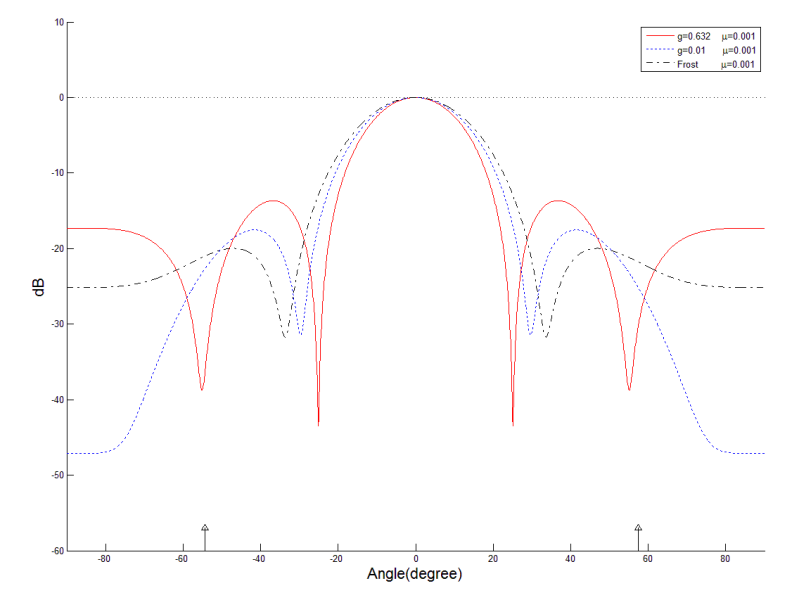

Fig. 9. Comparison of the beam patterns for the case of two coherent interferences.

\section{CONCLUSIONS}

A narrowband general linearly constrained adaptive array is examined in the weight vector space to calculate the array performance with respect to the gain factor in a coherent signal environment. It is observed that a variation of the gain factor results in a variation of the distance between the constraint plane and the origin in the translated weight vector space. This phenomenon has an effect on the nulling performance of the general linearly constrained adaptive array. Further, it is shown that the general linearly constrained adaptive array performs better than the linearly constrained adaptive array.

\section{ACKNOWLEDGMENTS}

This work was supported by the University of Incheon Research Grant, 2011.

\section{REFERENCES}

[1] O. L. Frost III, "An algorithm for linearly constrained adaptive array processing," Proceedings of the IEEE, vol. 60, no. 8, pp. 926935, 1972.

[2] T. J. Shan and T. Kailath, "Adaptive beamforming for coherent signals and interference," IEEE Transactions on Acoustics, Speech and Signal Processing, vol. 33, no. 3, pp. 527-536, 1985.

[3] V. U. Reddy, A. Paulraj, and T. Kailath, "Performance analysis of the optimum beamformer in the presence of correlated sources and its behavior under spatial smoothing," IEEE Transactions on Acoustics, Speech and Signal Processing, vol. 35, no. 7, pp. 927936, 1987.

[4] B. K. Chang, N. Ahmed, and D. H. Youn, "Fast convergence adaptive beamformers with reduced signal cancellation," in Proceedings of the 22nd Asilomar Conference on Signals, Systems and Computers, Pacific Grove, CA, pp. 823-827, 1988.

[5] B. K. Chang, C. H. Jeon, and D. H. Song, "Performance improvement in alternate mainbeam nulling by adaptive estimation of convergence parameters in linearly constrained adaptive arrays," International Journal of KIMICS, vol. 7, no. 3, pp. 392-398, 2009.

[6] B. K. Chang, T. Y. Kim, and Y. K. Lee, "A novel approach to general linearly constrained adaptive arrays," Journal of Information \& Communication Convergence Engineering, vol. 10, no. 2, pp. 108-116, 2012.

[7] B. Widrow and S. D. Stearns, Adaptive Signal Processing. Englewood Cliffs, NJ: Prentice-Hall, 1985.

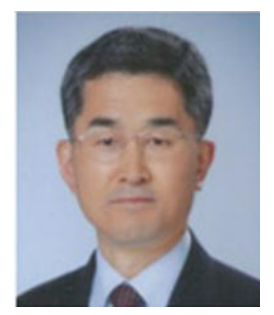

\section{Byong Kun Chang}

Dr. Chang received his B.E. degree from Dept. of Electronics Engineering, Yonsei University, in 1975, his MS degree from Dept. of Electrical and Computer Engineering, University of lowa, in 1985, and his Ph.D. degree from Dept. of Electrical and Computer Engineering, University of New Mexico, in 1991. Currently, he is Professor in the Dept. of Electrical Engineering at University of Incheon. His research interests include adaptive signal processing, array signal processing, and microcomputer applications. 